Voix et Images

\title{
Les carnets d'écriture inédits de Marie-Claire Blais
}

L'espace d'une oeuvre en gestation

\section{Marie-Claire Blais's Unpublished Notebooks. The Space of a \\ Work in Progress \\ Los cuadernos de escritura inéditos de Marie-Claire Blais. El espacio de una obra en gestación}

\section{Julie Leblanc}

Volume 37, numéro 1 (109), automne 2011

Marie-Claire Blais

URI : https://id.erudit.org/iderudit/1006460ar

DOI : https://doi.org/10.7202/1006460ar

Aller au sommaire du numéro

Éditeur(s)

Université du Québec à Montréal

ISSN

0318-9201 (imprimé)

1705-933X (numérique)

Découvrir la revue

Citer cet article

Leblanc, J. (2011). Les carnets d'écriture inédits de Marie-Claire Blais : l'espace d'une oeuvre en gestation. Voix et Images, 37(1), 31-43.

https://doi.org/10.7202/1006460ar
Résumé de l'article

Les carnets d'écriture inédits de Marie-Claire Blais nous donnent accès a sa personnalité créatrice, à ses dessins artistiques et à sa posture intellectuelle vis-à-vis de l'oeuvre en gestation. Ils ont une valeur génétique indéniable, car ils représentent une couche de manuscrits, voire un ensemble d'autographes préparatoires au sein desquels se trouve étalée l'histoire de la genèse de nombreux romans en voie de production. Les Carnets privilégiés dans cette étude, à savoir ceux qui sont contemporains à la rédaction d'Une saison dans la vie d'Emmanuel, de Testament de Jean Le Maigre à ses frères et de David Sterne servent à enregistrer des notes d'idées et de projets et à donner consistance à la fiction qu'il faut écrire. Espace de toutes les libertés, les Carnets de Blais sont également saturés de schémas, de plans illustrés et de portraits peints et dessinés de la main de l'auteure. Ces manuscrits nous permettent de retracer sur les plans textuels (de l'étape prérédactionnelle à l'étape rédactionnelle) et visuels (des schémas aux portraits, en passant par les croquis) la lente et progressive élaboration de ces trois romans. C'est en cernant les tâtonnements de l'écriture, les pulsions soudaines du tracé (croquis et portraits), que la vie de cette production littéraire et iconographique en mouvement a pu être restituée. 


\section{LES CARNETS D'ÉCRITURE INÉDITS DE M A R IE-CL A I R E B L A IS \\ $L^{\prime}$ espace d'une œuvre en gestation *}

$++$

JULIE LEBLANC

Université de Toronto

La forme graphique redouble le sens du mot;

verbal et non verbal disent la même pensée ${ }^{1}$.

Je propose d'aborder les thèmes qui me retiendront dans cette brève étude des Carnets $^{2}$ de Marie-Claire Blais, notamment celui de l'aventure des formes en devenir, des facettes protéiformes de leur avènement, en adoptant une approche génétique. Le work in progress, pour reprendre la formule de James Joyce, permet d'observer le sujet dans ses actes et privilégie ainsi la question du sujet de l'écriture. Cette perspective m'incite tout naturellement à problématiser la relation entre l'acte d'écriture, le sujet écrivant et le texte en gestation. Les manuscrits d'un écrivain, voire ses avanttextes, offrent une vision directe des traces matérielles d'une écriture en cours d'élaboration. C'est sur ce plan que les Carnets de Marie-Claire Blais sont indispensables à la saisie du mécanisme d'engendrement du texte en voie de production. Depuis que la critique génétique s'intéresse aux manuscrits littéraires avec de nouvelles exigences et de nouveaux outils méthodologiques, elle ne se restreint plus aux brouillons et aux différents états manuscrits des œuvres, mais s'ouvre aussi aux carnets de travail, aux cahiers et aux journaux des écrivains. Les Carnets de Blais donnent à voir le surgissement d'une forme ou d'une pensée, l'effet d'un événement, le détail du quotidien aussi bien que l'essentiel des découvertes narratives. Ces

$$
+++
$$

* Pour obtenir la liste des sigles utilisés dans cet article et les références complètes aux œuvres de Marie-Claire Blais, voir p. 7. 1 Louis Hay, La littérature des écrivains. Questions de critique génétique, Paris, José Corti, coll. «Les essais», 2002, p. 198. 2 Voici la description matérielle de ces carnets, tous tirés de Bibliothèque et Archives Canada : fonds Marie-Claire Blais/LMS-0117/Acquisition 1985-06 : Carnet II (Noël 1962 à mai 1963 ; janvier 1965 à avril 1965) : 144 p., croquis de visages à la dernière page ; Carnet III (avril 1965 à août 1965) : 142 p., dix-huit aquarelles ou pastels (décorations) en bordure de page, quinze petites aquarelles collées et trois grandes aquarelles collées; Carnet V (décembre 1965 à janvier 1966) : 136 p., dix grandes aquarelles collées, treize petites aquarelles collées, plusieurs photographies originales de paysages, deux portraits et quelques dessins; Carnet VII (juillet à novembre 1966) : 118 p., réflexions à la suite de rencontres, d'événements, de films, etc., trente-six aquarelles; Carnet VIII (1966-1967) : 114 p., réflexions, descriptions de rêves, quarante-huit aquarelles. (http://collectionscanada.gc.ca/pam_archives/index.php?fuseaction=genitem.displayItem\&lang=fra\&rec_nbr=3671391\&rec_nbr_list =3671391,106869,106783,206340,205881 [page consultée le 4 octobre 2011]) 
documents nous permettent d'observer la création littéraire «bien avant que l'écriture se fasse texte, parfois même avant qu'un projet d'œuvre soit né ${ }^{3}$ ». L'apport original de la critique génétique à l'œuvre de Marie-Claire Blais sera mis en évidence par la description des Carnets, du rôle que jouent les schémas, les plans de travail accompagnés de croquis et les portraits peints de la main de l'auteure. L'approche adoptée permet de donner un sens et une fonction à des documents demeurés muets pendant des décennies.

\section{LA SPÉCIFICITÉ GÉNÉTIQUE DES CARNETS}

C'est par leur identité matérielle que ces Carnets se distinguent des autres documents conservés dans les fonds Marie-Claire Blais de Bibliothèque et Archives Canada et de Bibliothèque et Archives nationales du Québec ${ }^{4}$. Il s'agit de cahiers reliés, dont les entrées datées donnent une certaine séquentialité aux centaines de pages qui composent l'ensemble de ces documents autographes. Les journaux d'écriture qui seront étudiés ici comprennent plusieurs carnets qui portent sur la genèse des trois textes ${ }^{5}$ romanesques que j'ai privilégiés et qui peuvent être classés chronologiquement en fonction des rédactions auxquelles ils se rapportent directement. Ce lieu de réflexion, où se dénoue l'histoire d'un texte en gestation, où le récit trouve sa forme et sa matière, permet de rendre compte des différentes opérations narratives et descriptives qui alimentent le sujet écrivant. Les Carnets de Blais contemporains de la genèse d'Une saison dans la vie d'Emmanuel, de Testament de Jean Le Maigre à ses frères et de David Sterne sont essentiels pour comprendre l'œuvre et surtout le processus global dont elle résulte. Les moments successifs de la rédaction sont bien enregistrés, de la phase prérédactionnelle (provisionnelle, exploratoire, préparatoire, d'initialisation) à la phase rédactionnelle (scénarique, documentaire, structurelle, textuelle). Autrement dit, ces Carnets témoignent du travail de conception préliminaire sous forme de plans, de notes, de scénarios, de résumés et d'esquisses ayant pour fonction de préparer et d'organiser la rédaction des différents romans en question. Il s'agit d'un véritable journal-atelier, d'un journal d'écriture, d'un laboratoire fécond pour Blais, qui se sert non seulement de cet espace pour recueillir ses projets littéraires, mais aussi pour dessiner et peindre des croquis et des portraits. Les Carnets se rapportent directement à la rédaction des romans: structures narratives, thèmes exploités, mises en œuvre des personnages et des enjeux socioculturels. À l'instar d'un journal personnel, ces Carnets sont saturés d'interventions auctoriales indiquant la posture intellectuelle et affective

3 Louis Hay, «L'amont de l'écriture», Louis Hay (dir.), Carnets d'écrivains I, Paris, Éditions du CNRS, 1990, p. 3. 4 Les carnets inédits de Marie-Claire Blais, notamment les Carnets II à XII sur lesquels je travaille depuis dix ans, ont été photographiés, transcrits, annotés et analysés par mes soins. Ils seront publiés par les Éditions du GREF et par Les Éditions du remue-ménage. 5 Ces Carnets portent sur la genèse de trois romans: Une saison dans la vie d'Emmanuel, David Sterne et Testament de Jean Le Maigre à ses frères. Bien que ce dernier soit un roman inachevé et inédit, deux fragments en ont été tirés pour publication : «La nouvelle institutrice», Les Lettres nouvelles, décembre 1966-janvier 1967, p. 88-100; «Testament de Jean-Le Maigre à ses frères ", Liberté, vol. XIV, nº 3, maijuin 1972, p. 74-83. 
Carnet II, sur Une saison dans la vie d'Emmanuel:

Écrire un livre où n'y aurait rien que ce clair dépouillement plein de choses dont je rêve. L'océan, le ciel, un homme seul, et ses pensées les plus simples et les plus belles. Un livre comme une prière [...]. Très difficile, ce moment éclairé et sombre, où Héloïse veut franchir l'échec de sa misérable vie, en quelque sorte. Je continuerai demain. [...] Je veux aussi parler davantage de Pomme et du Septième, et de leur apprentissage à la ville. Je dois d'abord y penser beaucoup [...] de nombreuses erreurs d'imagination, etc. [...] Alexis qui refuse le progrès "pas d'électricité dans la maison grand-mère». Sa domination aveugle des autres (surtout les femmes, sa mère, sa grand-mère). Sa ressemblance de plus en plus marquée avec le père [...] L'esprit de caste pour le Septième. Les ouvriers. L'immigrant à la valise. [...] Alexis qui regarde les moissons avec inquiétude [...]. Refus de Dieu.

\section{Carnet III, sur Testament de Jean Le Maigre à ses frères:}

À nouveau la crainte de manquer ce livre [...]. Il faudra penser à cela - chasser la peur, vivre de ce pauvre équilibre, de cette mince justification que l'on se crée chaque jour. Peut-être commencer à partir du chapitre Judith Prunelle [...] faire intervenir les personnages du passé mais avec une grande discrétion - Ne pas trop séparer les portraits mais ne pas trop les unir non plus.

\section{Carnet VII, sur David Sterne:}

David Sterne [...] le cauchemar de ce livre [...]. C'est direct et cruel, simple et barbare comme le personnage. Ce livre m'envahit complètement - et peut-être est-ce nécessaire ainsi? Il est vrai que tout idéal doit être détruit, tout héros renié, tout maître trahi, si l'on veut vivre [...]. Ne pas se laisser toucher par la façon américaine de voir les choses - américaines - car c'est la voix du maître [...]. Ce maître exige, montre, paraît étrange - insupportable [...]. Je rencontre un homme dans la rue à qui je parle des horreurs de la guerre du Vietnam «I am writing a diary, called the 50 days of the Vietnam war» - lui aussi est d'accord que cette guerre est horrible, inutile [...]. Ce soir inquiétude folle apprenant à la TV que l'on avait encore bombardé Hanoï — Voilà le présent, l'avenir! À quoi sert la lutte!

6 Ayant obtenu une bourse Guggenheim en 1963, Marie-Claire Blais s'est installée aux États-Unis pendant de nombreuses années. Les carnets inédits qui font l'objet de mon étude ont été rédigés lorsqu'elle y séjournait. Pendant toute l'année 1967, les bombardements se poursuivent au Vietnam. En 1968, les pertes américaines sont supérieures à cent morts par semaine. Les années 1967 et 1968 sont marquées par des émeutes raciales, et des milliers de citoyens américains s'insurgent contre la guerre. 
On voit à partir des extraits cités qu'il est possible de traiter les manuscrits prérédactionnels des romans comme le «récit d'une genèse » avec sa propre instance narrative (Marie-Claire Blais), ses actants, son contenu événementiel, ses péripéties, ses descriptions et son cadre: «Écrire un livre où n'y aurait rien que ce clair dépouillement plein de choses dont je rêve» ; "de nombreuses erreurs d'imagination », "à nouveau la crainte de manquer ce livre [...]. Il faudra penser à cela»; «le cauchemar de ce livre»; "ce livre m'envahit»; "peut-être est-ce nécessaire». La relation auteur/ manuscrit est «radicalement» autant que "paradoxalement existentielle ${ }^{7}$ ». Blais vit au sein de tensions sociopolitiques qui la perturbent énormément: «je parle des horreurs de cette guerre du Vietnam [...] horrible et inutile [...] à quoi sert la lutte!» Elle est aussi témoin des émeutes raciales (qui frôlent la guerre civile dans de nombreux États américains), des mouvements anti-guerre. Par ailleurs, si le texte achevé porte la signature de l'auteur, l'énonciateur, lui, ne survit réellement que dans les dossiers préparatoires où il manifeste sa présence par des "emplois performatifs » du «je ${ }^{8}$. Les nombreuses expressions de modalité qui parsèment les entrées des Carnets consacrées à la rédaction des romans donnent l'impression que la genèse des différentes composantes du récit est issue d'un enchaînement de volontés (vouloir-faire) : «je veux»; de nécessités (devoir-faire): «je dois», «tout idéal doit être détruit»; de possibilités (pouvoir-faire) : «il faudra penser à cela»; et de compétences (savoirfaire). On trouve aussi plusieurs expressions qui trahissent les hésitations de Blais quant aux différentes composantes de l'œuvre en cours: "peut-être commencer»; «peut-être est-ce nécessaire ainsi »; «il est vrai»; etc.

La lecture des plans, notes et scénarios donne l'impression d'une «lente

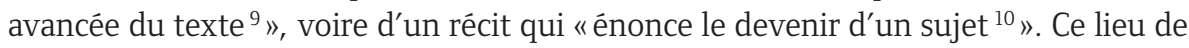
réflexion où le récit en gestation trouve sa forme et sa matière permet de rendre compte des diverses opérations narratives et discursives qui alimentent le sujet écrivant et sous-tendent les actes matériels et intellectuels de la créativité littéraire. Le métalangage qui parsème les Carnets est normalement axé sur le fonctionnement narratif du récit en voie de production. De cette conception auctoriale de la narrativité ressort la valorisation d'une des principales composantes de l'appareil narratif : la catégorie du personnage. Que celle-ci soit introduite à l'étape prérédactionnelle ou rédactionnelle, elle est traitée dans les Carnets comme un organisateur textuel, un lieu d'investissement du sujet et du social et un jalon du parcours isotopique du récit. La cooccurrence, pour ne pas dire la concurrence, de l'écrit et de la dimension graphique qui caractérise les premiers plans de travail met bien en évidence les personnages du roman en gestation.

7 Michel Contat, «Introduction. La question de l'auteur au regard des manuscrits», Michel Contat (dir.), L'auteur et le manuscrit, Paris, Presses universitaires de France, coll. "Perspectives critiques», 1991, p. 30.8 Almuth Grésillon, Éléments de critique génétique. Lire les manuscrits modernes, Paris, Presses universitaires de France, coll. «Poétique», 1994, p. 147. 9 Jacques Neefs, "Carnets de romanciers (Flaubert, Zola, James)», Littérature, n 80, décembre 1990, p. 63. 10 Claude Brémond, Logique du récit, Paris, Éditions du Seuil, 1973, p. 101. 


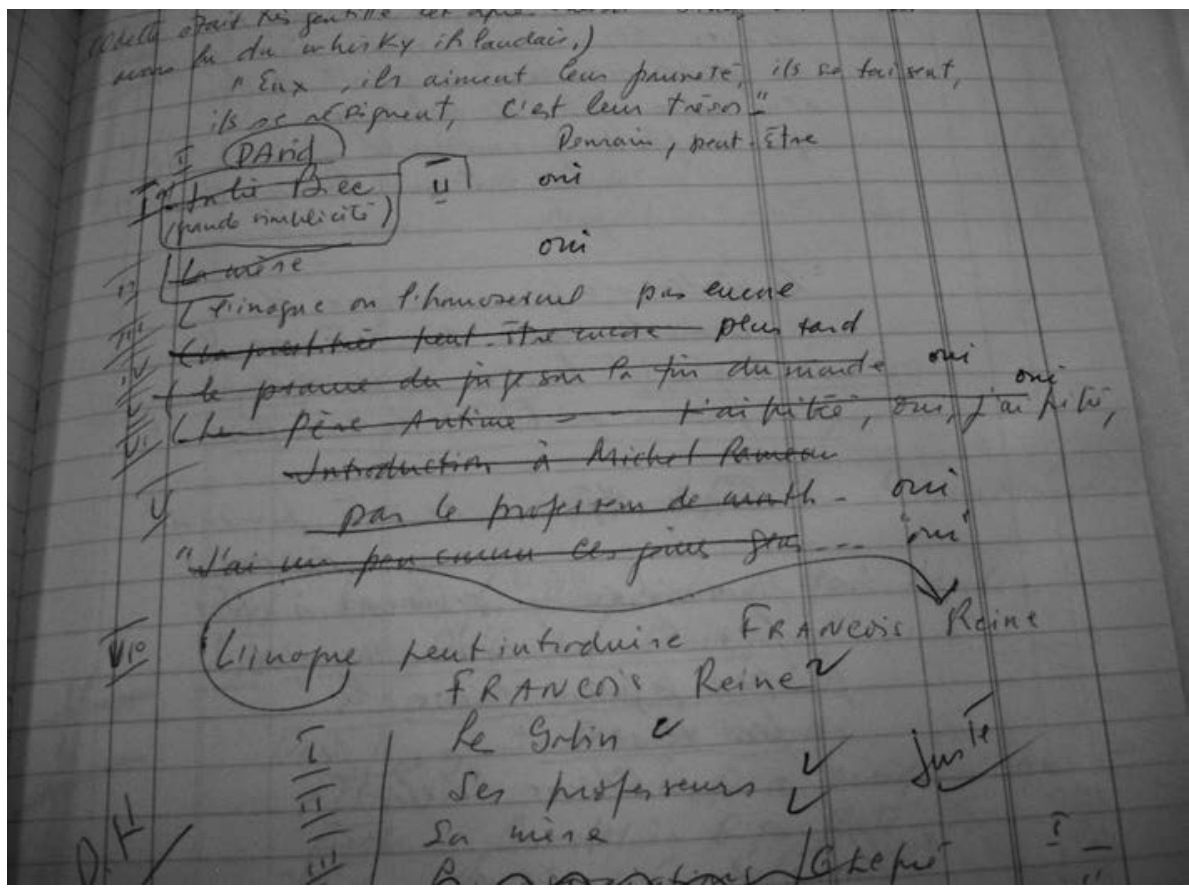

Figure 1 : Plan de David Sterne

Les plans préliminaires nous éclairent sur la genèse de l'œuvre et jouent un rôle stratégique pendant toute la durée de la rédaction. De fait, ces notes et plans sont les piliers d'une esthétique de la composition que l'on retrouve dans tous les Carnets de Blais consacrés à la rédaction d'Une saison, de Testament et de David Sterne. La manière dont les différentes composantes de l'architecture romanesque sont conçues et développées est non seulement révélatrice des modes de l'inventivité textuelle, mais aussi d'une certaine poétique narrative. Il est indéniable que l'écrivain sensibilisé à l'artisanat de la production littéraire fait tout naturellement appel au métalangage romanesque pour évoquer, décrire, analyser ses préoccupations scripturales. Les noms de personnages sont associés à des phénomènes temporels («le présent, le passé, le futur »), à la valeur symbolique des saisons («le printemps, l'été, l'hiver») et aux principaux leitmotive du roman : «la mort, le baptême, la résurrection; la pauvreté, la malnutrition, la déchéance ». D'énoncés purement descriptifs (Testament : «bonheur souffrance, inquiétudes, VIE; luxure, désespoir, désaccord, MORT») aux fragments appartenant à la phase textualisante (Testament: «J. L. Maigre, je l'ai bien reconnu [...] dommage qu'il soit mort»; David Sterne : «J'ai un peu connu ces jeunes gens... oui »), en passant par des unités de texte vouées à encadrer les différentes composantes actantielles du récit (Testament: «Judith, Abbé I. Innocence [...] Théodule, Jean Le Maigre, II. Luxure»; David Sterne : «l'ivrogne ou l'homosexuel, la prostituée, le juge, le professeur de math, l'ivrogne, les professeurs, sa mère : David, Julie, Michel, 
François »), les Carnets de Blais permettent d'élaborer une sorte de poétique génétique fondée sur les principales composantes de tout récit: le cadre de l'action, l'histoire racontée, les personnages et les thèmes qu'ils engendrent. Il n'est pas surprenant que les matériaux avant-textuels retrouvés dans les Carnets représentent un espace privilégié pour dégager l'ensemble des principes (stylistiques, thématiques et formels) qui régissent la structure du récit, la mise en œuvre des personnages et les données sociohistoriques et culturelles qui sous-tendent la rédaction d'Une saison, de Testament, et de David Sterne. Autrement dit, il est possible de dégager des Carnets une «syntaxe de la composition, de la logique et de la rythmique romanesques ${ }^{11}$ », toutes étroitement liées aux techniques de caractérisation des personnages.

Ce n'est qu'après avoir photographié, transcrit, organisé, annoté et analysé ces Carnets que j'ai constaté les similarités exceptionnelles qu'ils partagent. En dépit des différences flagrantes entre les trois textes sur les plans thématique et formel, Blais a toujours recours à des schémas et à des plans de travail accompagnés de croquis pour organiser de façon visible la matière brute et touffue du récit; les personnages, l'histoire et les thèmes sont représentés visuellement à cette étape de la genèse. À l'étape rédactionnelle, les schémas et les plans illustrés se métamorphosent en portraits peints et dessinés. Blais travaille selon le principe d'une "programmation scénarique». L'opposition entre une écriture à «structuration rédactionnelle» (qui ne s'appuie sur aucun schéma écrit, qui commence par une rédaction de premier jet et se construit au fil de l'élaboration de l’œuvre) et une écriture à « programmation scénarique » (qui fait précéder l'écriture par un travail de conception préliminaire sous la forme de plans, notes et scénarios) est importante car elle renvoie à deux façons de travailler. Préalablement à l'écriture d'un roman, Blais se consacre à un travail de réflexion au cours duquel elle utilise des schémas, des plans et des notes pour déterminer l'orientation de son projet d'écriture. Des semaines (Une saison et David Sterne) et même des mois (Testament) ont ainsi été consacrés à construire des canevas narratifs et iconographiques.

\section{LES PLANS ILLUSTRÉS}

Les plans de travail constituent une classe bien définie d'avant-textes d'une richesse génétique sans pareille. Leur multiplication dans les Carnets contribue à donner l'impression d'une genèse progressant par objectifs déterminés : «le testament est pur et sobre, le Septième maison de correction, prison pour vol, ivrogneries ». Tant pour Une saison que pour Testament et David Sterne, Blais réfléchit bien avant la rédaction aux personnages et à leurs actions, calcule les étapes successives du récit, prévoit la structure narrative du roman et enfin sa validité didactique. C'est aussi au cours de cette étape de la rédaction qu'apparaissent les plans illustrés qui confèrent une dimension

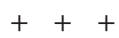

11 Henri Mitterand, "Genèse de La faute de l'abbé Mouret», Louis Hay (dir.), Les manuscrits des écrivains, Paris, Hachette/Éditions du CNRS, 1993, p. 203. Les expressions «structuration rédactionnelle» et "programmation scénarique» sont également empruntées à Mitterand. 


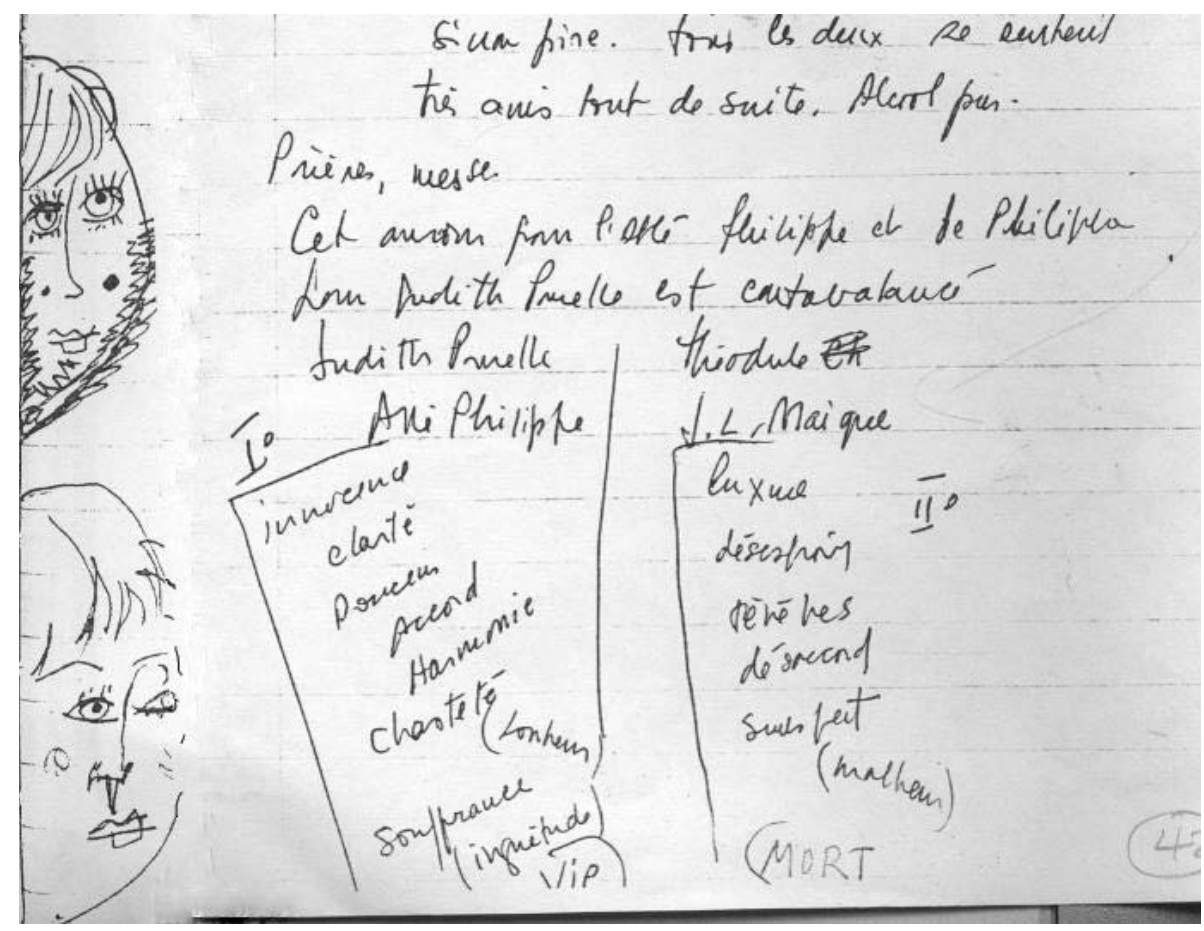

Figure 2: Testament de Jean Le Maigre à ses frères

iconographique aux Carnets $^{12}$. Je ne citerai ici que quelques exemples, mais ils sont suffisamment nombreux au sein des divers Carnets pour prouver leur prégnance au cours de la genèse des trois romans. Ce qui me paraît pertinent, c'est qu'à l'étape prérédactionnelle, les Carnets portant sur l'écriture d'Une saison, de Testament et de David Sterne présentent à l'évidence de nombreux traits communs, non seulement du point de vue de leurs règles de production, mais aussi du point de vue iconographique. En passant des plans sommaires, où sont disposés en schémas des substantifs nominaux décrivant la structure actantielle du récit, aux plans illustrés, qui présentent plus nettement les profils narratifs et diégétiques du roman, Blais apparaît comme un écrivain aux prises avec la «rationalité» de son texte ${ }^{13}$. Les plans du roman portent tous sur l'agencement événementiel de l'histoire racontée, sur les données spatiotemporelles du récit, de même que sur les personnages destinés à participer au parcours narratif déterminé.

12 La question des croquis introduits au sein des Carnets est abordée à la fin de l'étude d'Une saison dans la vie d'Emmanuel publiée dans mon ouvrage: Genèses de soi. L'écriture du sujet féminin dans quelques journaux d'écrivaines, Montréal, Les Éditions du remue-ménage, 2008, p. 73-102. Les rapports texte/image au sein des carnets inédits de Blais ont fait l'objet de deux projets soutenus par le CRSH (Conseil de recherches en sciences humaines du Canada) : «L'écriture autobiographique illustrée» (2006-2009) et « Genèses des rapports texte/image dans l'écriture diaristique de quelques écrivains et artistes» (2010-2013). 13 Cette expression est empruntée à Henri Mitterand, "Programme et préconstruit génétiques : le dossier de L'assommoir », Essais de critique génétique, Paris, Flammarion, coll. «Textes et manuscrits», 1979, p. 209. 
Quelle que soit la nature de l'unité textuelle témoignant des différentes phases génétiques auxquelles est soumise l'œuvre en cours (notes de régie, plans de travail, scénarios, résumés, esquisses, ébauches rédactionnelles) et quel que soit l'objet des remarques métalinguistiques présentées (la structure du récit, la voix narrative, l'organisation thématique du roman, le cadre spatiotemporel de l'histoire racontée), le personnage apparaît dans les cahiers de Blais comme le principal objet de discussion. La mise en œuvre des personnages et l'art du portrait manifestes au sein des Carnets revêtent différentes fonctions de l'ordre de la mimesis, du témoignage ou de la dénonciation. Une bonne approche de la notion du personnage dans les Carnets doit tenir compte des visées de l'auteure, des fonctions précises qui lui sont attachées, du contexte sociopolitique dans lequel il fut conçu et des rapports qu'entretient l'écrivaine avec la pensée et les idéologies de l'époque: l'ère de l'anticléricalisme, de la contestation des principales institutions sociales, de la guerre du Vietnam, des luttes raciales et des mouvements pacifistes américains.

Ces éléments premiers de textualisation accompagnés d'images (croquis et portraits) interviennent dans le processus littéraire, lui confèrent une dimension iconographique et mettent en place un nouvel imaginaire où l'écriture et le dessin se recoupent. Dans les Carnets, la genèse d'Une saison, de Testament et de David Sterne ne relève donc pas uniquement de l'écriture: les portraits (croquis, dessins, aquarelles) exécutés par Blais l'accompagnent au cours de sa rédaction et jouent un rôle important dans le processus de l'invention. Ils se glissent dans les Carnets pour exorciser les obsessions de l'écrivaine, frayer la voie à l'écriture, croquer les silhouettes des personnages, contribuer au développement du récit, illustrer une idée qui ne peut être concrétisée par les mots, et engendrer les suppressions et les ajouts auxquels a donné lieu cette insistance obsédante sur les préliminaires de l'écriture. L'art du portrait vient donc à la rescousse du processus créateur et renvoie lui-même à l'idée de genèse : qu'il s'agisse de leur description ou de leur représentation picturale, les personnages dans le texte et les avant-textes de ces trois romans sont un lieu important pour l'articulation des stratégies de lecture et d'écriture. L'écriture, la lecture et la réécriture des différentes composantes des romans - les personnages, l'histoire racontée, les thèmes et leur valeur didactique - sont bien mises en évidence dans ces documents avant-textuels. Les schémas préliminaires auxquels Blais fait appel pour organiser la matière romanesque donnent lieu à de nouveaux plans accompagnés de croquis. Ces derniers sont remplacés au cours de l'étape rédactionnelle par des portraits plus détaillés exécutés au stylo, au crayon ou à l'aquarelle. Cette dimension génétique des Carnets est extraordinaire, qui illustre l'évolution du texte et de l'image sur les plans textuels (de l'étape prérédactionnelle à l'étape rédactionnelle) et visuels (des schémas aux portraits, en passant par les croquis). Ces quelques extraits, destinés à mettre en évidence la complexité des rapports texte/image, fournissent non seulement une superbe illustration des échanges entre les réalités textuelles et figuratives que l'on retrouve dans les Carnets de Blais, mais proposent aussi aux études génétiques un domaine peu exploité. 
LES PORTRAITS PAINTS ET DESSINÉS

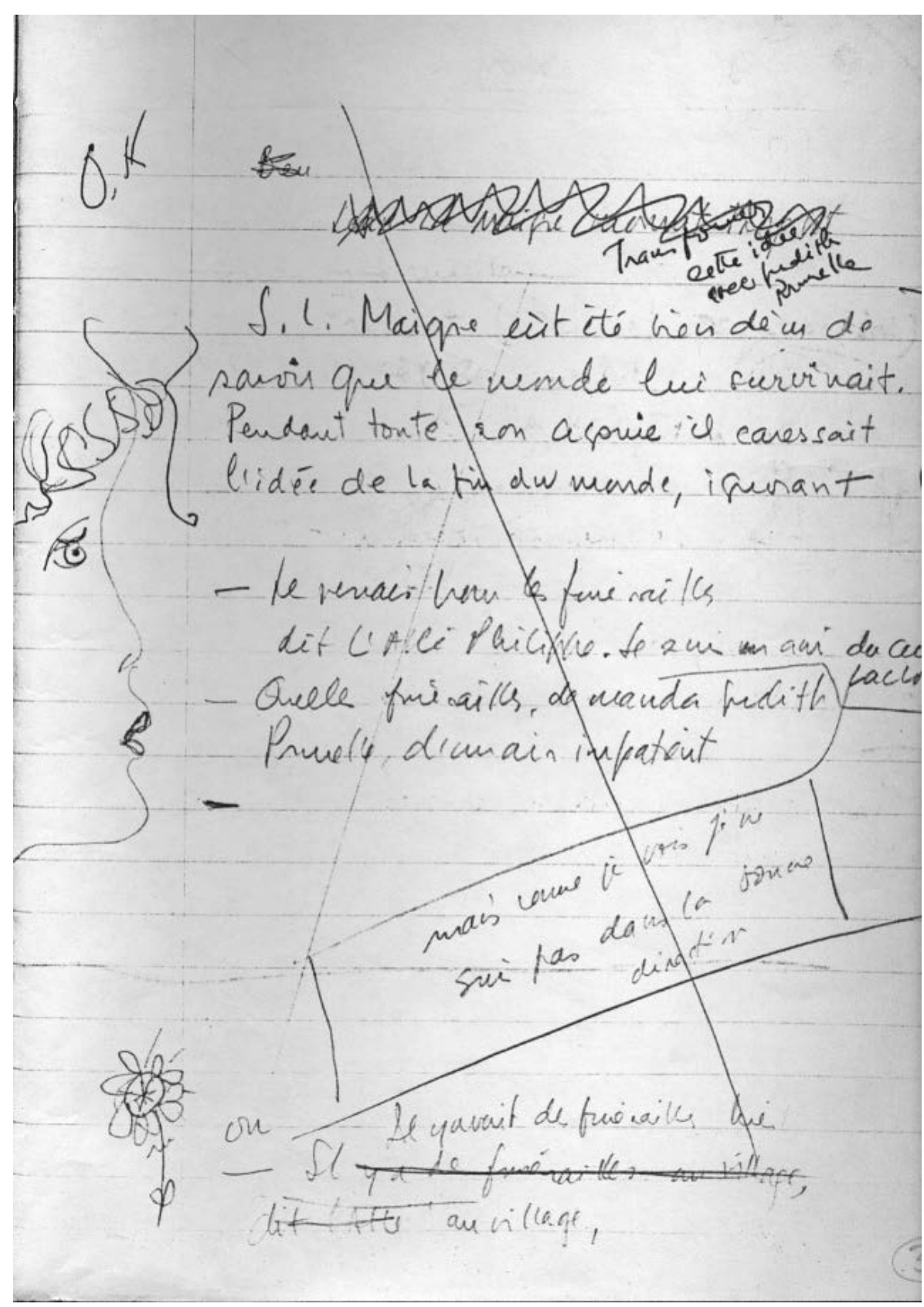

Figure 3 : Carnet V. Testament de Jean Le Maigre (scénario rédactionnel)

DOSSIER $\mathbf{3 9}$ 
Les portraits peints et dessinés apparaissent surtout dans les entrées des Carnets consacrées à la rédaction de Testament et de David Sterne.

Les Carnets de Blais sont, de fait, remplis de scénarios et d'esquisses partiellement rédigés. Quand le projet d'écriture commence à devenir un texte, en phrases amorcées, avec des subordinations syntaxiques et des verbes conjugués, on arrive à un autre stade de la création. Ces scénarios partiels sont le champ d'un immense travail qui prend en écharpe les autres phases répertoriées. À partir de quelques phrases, Blais se lance dans un court galop de rédaction directe. Le travail procède ainsi du général au particulier, d'une vue d'ensemble à un approfondissement des détails.

Seules les éventuelles reprises des notations autographes dans les rédactions successives de Testament et de David Sterne, notamment dans l'élaboration de la phase rédactionnelle, permettent d'accorder aux documents préparatoires retrouvés dans les Carnets leur fonction proprement textuelle et narrative. C'est en relisant les textes (publiés et inédits) à la lumière des observations, projections et spéculations que livrent les documents préparatoires introduits au sein des Carnets que l'on constate pleinement la valeur signifiante de la nature métadiscursive des documents autographes. Les considérations formelles qui animent les plans, les notes et les scénarios de ces romans trouvent une sorte de complétude dans les documents rédactionnels, en particulier dans les esquisses et les ébauches. En d'autres termes, les documents prérédactionnels de l'œuvre de Blais entretiennent d'étroits rapports avec la matière romanesque où sont textualisées certaines des observations, des spéculations et des projections énoncées dans les Carnets. De fait, le travail de conception narrative qui se dessine dans les plans, les notes de régie, les scénarios, les résumés, les esquisses et les ébauches déborde dans les tapuscrits inédits de Testament et dans le texte publié (David Sterne). Un des intérêts des Carnets de Blais provient précisément de cette proximité entre les matériaux prérédactionnels et rédactionnels, voire entre les plans, les scénarios et les esquisses:

Voici par exemple l'extrait tiré des tapuscrits inédits du Testament qui reprend de façon plus détaillée celui de la figure 3 :

$<$ R Il y a des funérailles au village, dit l'Abbé, $><$ A Il y avait des funérailles au village $<$ une phrase écrite un peu de travers, encadrée par un carré dessiné par Blais "parler de funérailles de cette manière ou autrement"> [R = rature; $A=$ ajout $]$

On m'a dit en route qu'il y a eu ce matin les funérailles d'un jeune frère. Il avait quatorze ans. Je ne sais pas son nom, dit l'Abbé Philippe. Je ne connais personne. Je vais remplacer le Curé Lacloche dans cette paroisse. Judith Prunelle regardait le prêtre avec surprise ${ }^{14}$.

On remarque que l'art du portrait auquel s'adonne Blais au cours de la rédaction de Testament et de David Sterne est étroitement lié à sa posture intellectuelle. Si les schémas et croquis qui illustrent les plans de travail constituent « une remontée aux

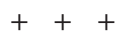

14 Le protocole utilisé est celui de la Bibliothèque du Nouveau Monde. 
sources ${ }^{15}$ ", les portraits marquent pour Blais un point culminant au cours de la rédaction et ont une autre signification dans la genèse de son œuvre. Les difficultés à décrire convenablement l'histoire de ses protagonistes (la souffrance et la mort de Jean Le Maigre et de David Sterne) ainsi que l'angoisse qu'éprouve Blais face à la politique américaine dominent les nombreuses entrées de ces Carnets : «le fait de vivre dans ce pays et d'avoir constamment sous les yeux, à la télévision, et dans les journaux, [c]e comportement aveugle (36 enfants tués - dans un village du Sud Vietnam)»; "dans le journal, il y a là un enfant à Hanoï victime d'un raid - c'est l'absurde, la destruction de la vie». Qu'il s'agisse du texte en cours (qui s'arrête au décès de ses personnages) ou de la guerre du Vietnam et de ses milliers de victimes, la mort apparaît comme le principal leitmotiv dans les Carnets consacrés à la rédaction de ces deux romans. Ces cahiers, qui portent sur la condition humaine, l'holocauste, les guerres nucléaires, sont ponctués de portraits peints et dessinés. Cette convergence de la littérature et du portrait est complexe, car il s'agit d'une véritable rencontre de formes et de sens. Dans certaines entrées des Carnets, les deux modes d'expression paraissent indissociables. Lorsque la figuration est incluse dans le graphisme, la rencontre du texte et de l'image prend la forme d'une création double (figures 2 et 3). Dans d'autres extraits, l'image double en permanence le récit - voir les figures 2 et 4 , ainsi que le portrait de Jean Le Maigre qui apparaît sur la couverture de la présente livraison de Voix et Images ${ }^{16}$.

Blais est parvenue à construire une situation dialectique où l'image intervient de façon active dans le processus de l'écriture. C'est grâce à cette alliance du mot et de l'image que circulent les informations sur les textes en gestation et sur les contextes socioculturels dont ils sont issus. Il ne faut pas oublier que l'analogie entre la mort et l'art du portrait est aussi ancienne que la fabrication des images elle-même. Dans l'histoire de l'art, le portrait trouve son véritable sens dans le fait de représenter quelque chose qui est absent/mort et qui peut donc seulement être là en image. La liaison symbolique qui s'instaure entre la pratique du portrait et la mort est une des constantes de ce genre pictural. Dans la mesure où les trois textes de Blais privilégient les thèmes de la souffrance, de la maladie et de la mort, il n'est pas surprenant que les Carnets consacrés à leur genèse soient aussi parsemés d'allusions à ce motif. Toutefois, le fait que Blais ait choisi les croquis et les portraits peints et dessinés pour représenter sur le plan iconographique son rapport intellectuel et affectif à l'œuvre en gestation est significatif : ces images entretiennent une dialectique insistante avec la pensée de la mort. Il s'agit de la mort des personnages, des personnes que Blais a connues au moment de la rédaction de ses romans ${ }^{17}$ et des victimes de cette guerre que Blais décrit à maintes reprises dans ses Carnets comme un «cauchemar»:

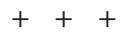

15 Louis Hay, La littérature des écrivains, p.189. 16 Ce portrait est présenté dans les Carnets au moment précis où Blais décide d'entamer la rédaction de David Sterne. L'art du portrait, notamment ceux de Jean Le Maigre dans les Carnets, a fait l'objet de deux de mes études: «Portraits écrits et portraits images dans les quelques journaux d'écrivains", Cécile Meynard (dir.), Les journaux d'écrivains. Enjeux génétiques et éditoriaux, Berne, Peter Lang, 2011, p. 50-172; "L'intensité du texte et de l'image», La Licorne, n 97, 2011, p. 179-181. 17 Dans Parcours d'un écrivain. Notes américaines, Blais précise que ce sont ses amis Jack et Robert à Cambridge qui lui ont donné l'idée d'écrire David Sterne: «Jack mon ami étudiant détruit par les drogues, Robert, le jeune 


W
O
$R$
$K$

I
$N$

\begin{abstract}
Carnet VIII
c'est ce poids qu'il y a une guerre interminable, injuste, chaque jour plus injuste, plus horrible l'on est là impuissant [...]. Et cela est mal - et cela est insupportable [...] avoir constamment sous les yeux, à la télévision, et dans les journaux, le comportement aveugle, se répétant sans même le savoir (36 enfants tués dans un village du Sud Vietnam) [...].

Rêve de l'homme évanoui - de la société américaine, de l'exil des Américains au cœur de leur monde aveugle. "It is not a dead man? " No just a sick man.» L'influence du mensonge dans le paradis. Les nouvelles ce matin à la radio évacuation de Hanoï [...] un génocide planifié et systématique non vraiment. Il ne peut y avoir d'avenir pour le monde, volontairement ou non, nous marchons vers notre fin. Les têtes malsaines ont décidé de la mort de nous tous. Leurs intentions sont si barbares qu'elles nous échappent. On ne peut pas supporter la tuerie continuelle. Si l'autre est assassin, toute la conscience du monde en souffre.
\end{abstract}

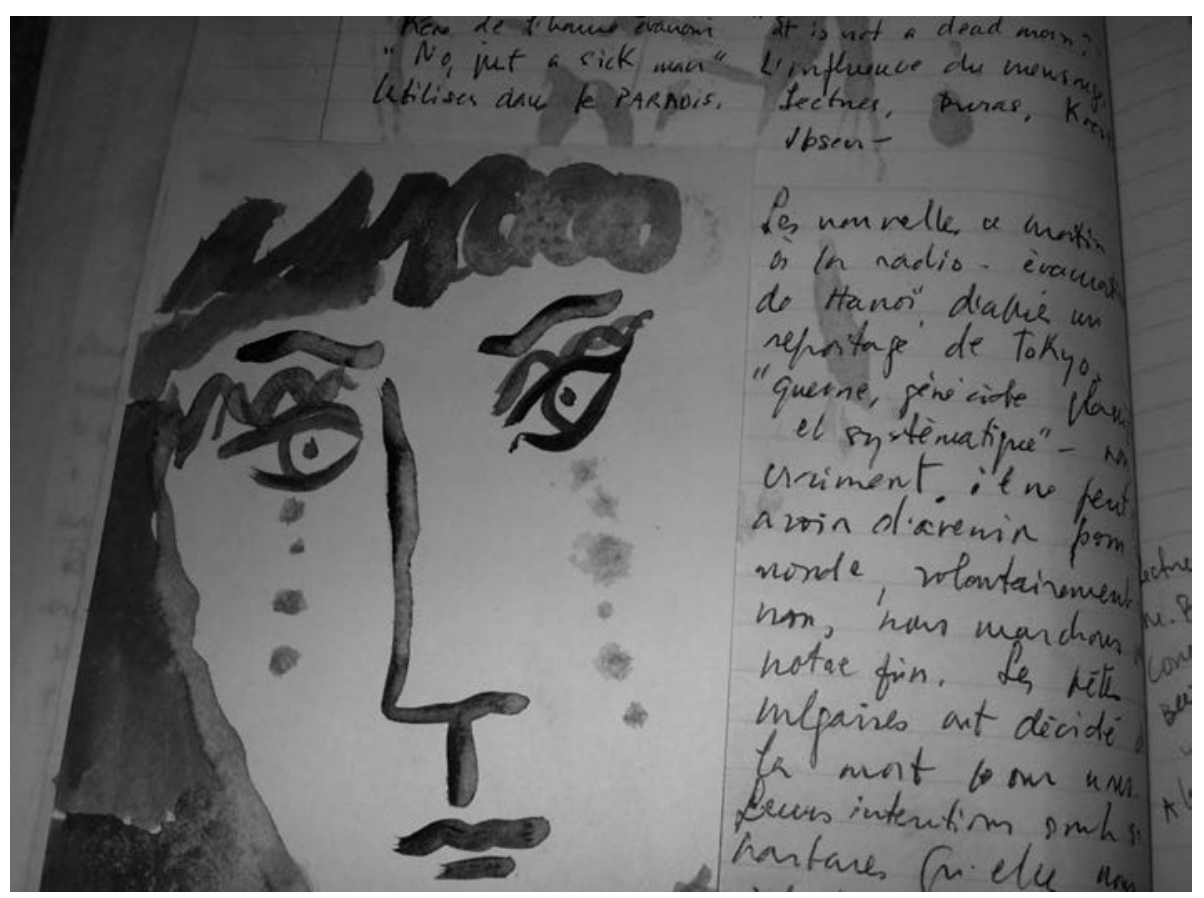

Figure 4: Portrait accompagnant l'extrait du Carnet VIII cité ci-dessus

$$
++
$$

écrivain noir meurtri à jamais par la cruauté du racisme [...]. Pendant que j'écris mon roman, les visages de Jack et Robert se confondent en une seule figure tragique, celle d'une génération vouée par les guerres, la guerre du Vietnam, la ségrégation qui a fait tant de victimes [...]» (PDE, 115-116). 
Ces observations attestent une circulation des sens entre le verbal et le pictural : l'un et l'autre sont les lieux d'un même conflit intérieur pour Blais. La description de son état d'âme face à la guerre du Vietnam donne lieu à ce triste portrait exécuté à l'aquarelle. On remarque également que certains des croquis et portraits renvoient directement à la mort (le décès de Jean Le Maigre, qui sert d'excipit à Une saison et d'incipit à Testament), tandis que d'autres y renvoient symboliquement: le portrait révèle l'absence. La production des images qui ponctuent les carnets inédits de Blais n'est jamais vide de signification.

\section{LA RICHESSE GÉNÉTIQUE ET ICONIQUE DES CARNETS}

Si les Carnets de Marie-Claire Blais constituent un remarquable objet d'étude, c'est non seulement parce qu'ils offrent un répertoire relativement complet des différentes phases génétiques, mais également parce qu'ils donnent accès aux différents romans en gestation. Par l'entremise de nombreux termes qui appartiennent au métalangage romanesque, on rencontre dans les Carnets une conception a priori du récit, en particulier de ses principales composantes : la structure narrative, l'articulation diégétique, l'histoire racontée et surtout la mise en texte des personnages. À l'instar de nombreux dossiers manuscrits, les Carnets de Blais donnent accès aux différentes stratégies d'écriture qui ont participé à la genèse d'Une saison, de Testament et de David Sterne. Par ailleurs, ils mettent bien en évidence l'investissement du sujet écrivant à l'étape prérédactionnelle. La manière dont les différentes composantes de l'architecture romanesque sont conçues et développées est non seulement révélatrice des modes de l'inventivité textuelle, mais aussi d'une certaine conception auctoriale de la production littéraire. À l'encontre d'autres écrivains (Annie Ernaux, Suzanne Lilar, Nicole Brossard, Madeleine Monette, Madeleine Ouellette-Michalska, Michel Leiris, Émile Zola, Georges Perec, etc.), dont les fonds de manuscrits se distinguent nettement d'un texte à l'autre, Marie-Claire Blais adopte les mêmes stratégies textuelles (plans, notes, scénarios, esquisses) et visuelles (schémas, croquis, portraits) d'un roman à l'autre. Dans la mesure où Blais passe plus ou moins directement de ses Carnets à une version dactylographiée de ses textes, c'est véritablement au sein de ce répertoire de documents autographes que la genèse de sa production littéraire s'effectue. Autrement dit, c'est dans cet espace d'écriture que réside la richesse génétique et esthétique des Carnets, que les contours de ces étranges objets de désirs périlleux, émouvants et multiformes (Une saison, Testament et David Sterne) se laissent entrevoir et approcher d'assez près. L'écriture de Blais déborde de toutes parts la linéarité du code et se projette dans des espaces multiples qui ne sont pas uniquement d'ordre linguistique, mais aussi d'ordre iconographique. Ce mariage entre le texte et l'image a permis à MarieClaire Blais de dédoubler les systèmes de significations, de multiplier les réseaux de lecture et de créer une relation dialectique extraordinaire entre l'écriture et l'image. Les Carnets de Blais viennent ainsi se ranger parmi ces écrits constituant un vaste laboratoire artistique qui se transforme peu à peu en expérience d'une écriture vécue. 


\section{IS T E DES S I G LES}

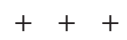

Afin d'alléger le système de notes, les références aux œuvres de Marie-Claire Blais mentionnées dans ce dossier seront indiquées par des sigles, suivis du folio, et placées entre parenthèses dans le texte:

Augustino et le chœur de la destruction (ACD), Montréal, Boréal, 2005, 302 p.

Dans la foudre et la lumière (DFL), Montréal, Boréal, 2001, 251 p.

Mai au bal des prédateurs (MBP), Montréal, Boréal, 2010, 322 p.

Manuscrits de Pauline Archange (PA), Montréal, Stanké, 1988 [1968], 223 p.

Manuscrits de Pauline Archange (MPA) [1968], Vivre! Vivre! (VV) [1969], Les apparences (LA) [1970], Montréal, Boréal, coll. «Compact», 1991, 324 p.

«Marie-Claire Blais», Catherine Morency (dir.), La littérature par elle-même (LPE), Québec, Nota bene, 2005, p. 17-21.

Naissance de Rebecca à l'ère des tourments (NR), Montréal, Boréal, 2008, 296 p.

Parcours d'un écrivain. Notes américaines (PDE), Montréal, VLB éditeur, 1993, 222 p.

Soifs (SO), Montréal, Boréal, 1995, 314 p.

Soifs (S), Montréal, Boréal, coll. «Compact», 1997 [1995], 320 p.

Une saison dans la vie d'Emmanuel (SVE), Montréal, Éditions du Jour, 1968 [1965], $128 \mathrm{p}$. 